\title{
Possibility Of THz Donor Lasing In Electrically Pumped Silicon
}

\author{
Roman Kh. Zhukavin ${ }^{\mathrm{a}}$, Veniamin V. Tsyplenkov ${ }^{\mathrm{a}}$, Valery N. Shastin ${ }^{\mathrm{a}}$, Sergey G. Pavlov ${ }^{\mathrm{b}}$, \\ and Heinz-Wilhelm Hübers ${ }^{\mathrm{b}, \mathrm{c}}$ \\ ${ }^{a}$ Institute for Physics of Microstructures RAS, 603950, Nizhny Novgorod, Russia \\ ${ }^{\mathrm{b}}$ Institute of Planetary Research, German Aerospace Center (DLR), 12489 Berlin, Germany \\ ${ }^{c}$ Institut für Optik und Atomare Physik, Technische Universität Berlin, 10623 Berlin, Germany
}

\begin{abstract}
A possible way to create silicon terahertz laser under electric field excitation is presented. Electrical pulses with both period and duration in nanosecond range should be applied to moderately doped stressed bulk silicon. The purpose of short pulse excitation is impurity breakdown followed by capture and population of upper lasing state. The mechanisms responsible for population inversion and losses are described.
\end{abstract}

\section{INTRODUCTION AND BACKGROUND}

$\mathrm{T}$ ERAHERTZ (THz) silicon lasers are based on impurity transitions of the hydrogen-like donor in monocrystalline silicon ${ }^{1}$. These lasers operate at low lattice temperatures under optical pumping by a mid-infrared laser and emit at frequencies between $1.2 \mathrm{THz}$ and $6.9 \mathrm{THz}$. Population inversion is achieved due to phonon-assisted relaxation in donor atoms ${ }^{2}$. Second mechanism of donor lasing is based on electronic Raman transitions. By tuning the pump laser frequency, the frequency of Raman laser can be tuned from 4.5 to $6.4 \mathrm{THz}^{3}$.

For majority of applications electrical pump is needed. First proposal for impurity based lasing under pulsed electrical field was published long ago ${ }^{4}$. We will present results on analysis of experimental results obtained for silicon lasers under intracenter optical pumping, stress experiments, measurements of terahertz losses and make estimations of possible gain and losses for electrically pumped silicon doped by shallow donors.

\section{RESULTS}

Application of continuous electric field results in impurity ionization and lasing mechanism based on accumulation of electron on long-lived excited state at cryogenic temperatures becomes broken. To overcome such difficulty short pulse excitation looks promising. Additionally, it has been demonstrated that at some stress silicon has much lower pump threshold due to longer lifetime, lower losses on $\mathrm{D}^{-}$centers and redistribution of the electrons over valleys. Typical photon lifetime in resonator of silicon lasers measured with use of short FEL pulsed excitation is about 10 ns. Taking this value as a starting point we put the limits for period of electrical field needed for excitation of the system equal to $10 \mathrm{~ns}$. Capture time for donors can be as fast as $0.1 \mathrm{~ns}$ for compensated crystals. So for purpose of simplicity we can suppose that decrease of current in the conduction band depends on electrical matching for the sample with resistance varying from small values under breakdown and big values for captured electrons. The modern possibilities of electronics give the chance to govern the current with speed about $1 \mathrm{GHz}$, so we take such a value for further estimates. Typical breakdown field is about $0.5 \mathrm{kV} / \mathrm{cm}$ for microsecond duration and should be higher for nanoseconds, that can be achieved by modern switches. Then we should compare gain and losses per period of excitation. Taking into account lifetimes of donor states to be several hundred picoseconds under the stress and using simple condition for amplification $\alpha \tau>\gamma \mathrm{T}$ ( $\alpha$ - gain, $\tau$ - lifetime of upper laser state, $\gamma$ - losses, $\mathrm{T}$ - period of excitation) we can estimate e.g. maximum suitable losses with given other parameters to be about $0.1 \mathrm{~cm}^{-1}$ (for $\alpha=1 \mathrm{~cm}^{-1}$, $\tau=500 \mathrm{ps}, \mathrm{T}=5 \mathrm{~ns}$ ). Such low value of losses nevertheless can be achieved for silicon with concentration about $10^{15} \mathrm{~cm}^{-3}$ and frequency about $5 \mathrm{THz}$ if taking into account only free carrier absorption. Figure presents illustration of pulsed pumping of silicon doped by donors assuming instant excitation, capture governed by acoustical phonon-assisted relaxation and losses measured for intracenter excitation $\left(0.01 \mathrm{~cm}^{-1}\right)$.

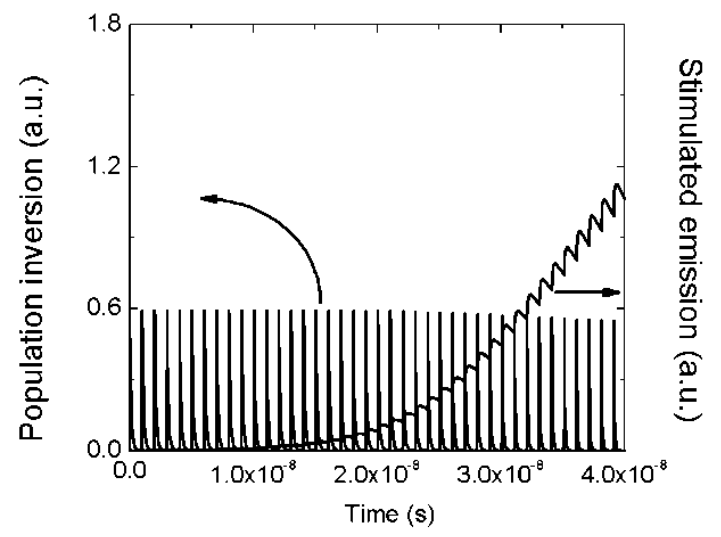

This work was partly supported by Russian Foundation for Basic Research (RFBR grants 11-02-00957 and 13-02-97116).

\section{REFERENCES}

[1] S. G. Pavlov, R. Kh. Zhukavin, V. N. Shastin, H.-W. Hübers, "The physical principles of terahertz silicon lasers based on intracenter transitions", Phys. Stat. Sol. b., vol. 250, 9-39 (2013) and refs therein.

[2] S. G. Pavlov, R. Kh. Zhukavin, E. E. Orlova, V. N. Shastin, A. V. Kirsanov, H.-W. Hübers, K. Auen, and H. Riemann, "Stimulated Emission from Donor Transitions in Silicon", Phys. Rev. Lett. vol. 22, 5220-5023 (2000).

[3] S. G. Pavlov, H.-W. Hübers, J. N. Hovenier, T. O. Klaassen, D. A. Carder, P. J. Phillips, B. Redlich, H. Riemann, R. Kh. Zhukavin, and V. N. Shastin, Stimulated Terahertz Stokes Emission of Silicon Crystals Doped with Antimony Donors, Phys. Rev. Lett., vol. 96, 037404 (2006).

[4] N.G. Basov, O.N. Krohin, Yu.M. Popov, "Generation, amplification and receiving of IR and visible radiation by quantum systems", Soviet Physics-Uspekhi, vol. LXXII, 162 (1960), in Russian. 